\title{
Novel Embryoid Body-Based Method to Derive Mesenchymal Stem Cells from Human Embryonic Stem Cells
}

\author{
Eun Ju Lee, Ph.D., ${ }^{1, \star}$ Ha-Neul Lee, M.S., ${ }^{1, \star}$ Hyun-Jae Kang, M.D., ${ }^{1,2}$ Keum-Hyun Kim, M.S., Jin Hur, Ph.D., \\ Hyun-Jai Cho, M.D., ${ }^{1,2}$ Jaewon Lee, B.S., Hyung-Min Chung, Ph.D., Jaejin Cho, D.V.M., Ph.D., ${ }^{4}$ \\ Mee-Young Cho, Ph.D., ${ }^{4}$ Sun-Kyung Oh, Ph.D., ${ }^{5}$ Shin-Yong Moon, M.D., \\ Young-Bae Park, M.D., ${ }^{1,2}$ and Hyo-Soo Kim, M.D., ${ }^{1,2,6}$
}

Application of human embryonic stem cells (hESCs) to stem-cell therapy is not feasible because of the risk of tumorigenicity and rejection. In contrast, human mesenchymal stem cells (hMSCs) are free from the risk of tumorigenicity and also have immune privilege. However, hMSCs obtained from adults have infinite variety in terms of the biological characteristics and functionality. We report here a new derivation method of hMSCs from hESCs. The derivation of hMSCs from three different hESC lines (SNUhES3, CHA3-hESC, and H9) was performed by embryoid bodies formation and subsequent culture with stage-different media without using inductive xenogenic feeder and mechanical selection procedure. The derived cells were morphologically similar to the unique fingerprint-like pattern of hMSCs and grew stably for at least 35 passages in vitro. These cells had hMSCs-like immunophenotypes: negative for CD34 and CD45; positive for CD29, CD44, CD73, CD90, and CD105. They could be differentiated into multiple lineages including osteocytes, chondrocytes, adipocytes, and myocytes. They maintained normal karyotype during the long-term cultivation and did not show tumorigenicity when transplanted into the immunodeficient mice. In conclusion, the new embryoid body-based derivation method of hMSCs from hESCs is simple, safe, and reproducible in three different hESC lines. We expect that this method will provide a more effective and powerful tool to derive hMSCs from various hESC lines.

\section{Introduction}

$\mathbf{S}$ TEM-CELL THERAPY is a promising treatment strategy for degenerative disease. However, current stem-cell therapy using human adult stem cells has several serious problems. First, the human adult stem cells have limited differentiation potentials and self-renewing capacities. The human adult stem cells were influenced by aging and diseases that the donors have suffered from. ${ }^{1}$ Additionally, harvest of stem cells in human adult tissue frequently requires invasive procedures that can limit applicability and supply of stem cells. Finally, there are wide varieties in the biological characteristics and functionality of the harvested human adult stem cells.

Human embryonic stem cells (hESCs) can be considered as an alternative to overcome the limitations of human adult stem cells. hESCs are believed to be more versatile than tissue-specific human adult stem cells. However, in terms of purity and yield, the current differentiation methods of hESCs are still unsatisfactory, even though it requires lots of complicated manipulations. $^{2-4}$ Additionally, there are concerns about the risks of tumor formation and immune rejection after transplantation. ${ }^{5,6}$ In contrast to hESCs, human mesenchymal stem cells (hMSCs) have immune privilege ${ }^{7}$ and are relatively free from the risk of tumorigenicity. If simple and high purity differentiation methods can be developed, derivation of hMSCs from hESCs will be an attractive platform for stem-cell therapy.

Recently, different groups have reported hMSC derivation from hESCs (hESC-MSC). ${ }^{8-10}$ However, these reported methods have the problems for the fine clinical application, such as the employment of animal feeder cells, limitation in

\footnotetext{
${ }^{1}$ National Research Laboratory for Cardiovascular Stem Cells and IRICT, Seoul National University Hospital, Seoul, Republic of Korea.

${ }^{2}$ Department of Internal Medicine, Seoul National University College of Medicine, Seoul, Republic of Korea.

${ }^{3} \mathrm{CHA}$ Stem Cell Institute, Pochon CHA University, Seoul, Republic of Korea.

${ }^{4}$ Lab of Developmental Biology and Stem Cell Differentiation/Transplantation, School of Dentistry, Seoul National University, Seoul, Republic of Korea.

${ }^{5}$ Medical Research Center, Institute of Reproductive Medicine and Population, Seoul, Republic of Korea.

${ }^{6}$ Ischemia/Hypoxic Disease Institute, Seoul National University College of Medicine, Seoul, Republic of Korea.

*These two authors equally contributed to this work.
} 
functional differentiation of derived cells, and the risk of cell damage induced by sorting process. ${ }^{8-10}$

In this study, we developed a novel derivation method for the multipotent hMSCs from hESCs based on the formation of embryoid bodies (EBs) and stage-different media without mechanical selection and xenogenic feeder cells.

\section{Materials and Methods}

\section{Culture of hESCS}

Three different hESC lines were used in this study: SNUhES3 (Institute of Reproductive Medicine and Population, Medical Research Center, Seoul National University, Korea), ${ }^{11}$ CHA3-hESC (Stem Cell Research Laboratory, CHA Stem Cell Institute, Pochon CHA University, Korea), ${ }^{12}$ and H9 (Wisconsin Regional Primate Research Center, University of Wisconsin). Mitomycin-C-treated (Sigma, St. Louis, $\mathrm{MO})$ SIM mouse embryo-derived thioguanine and ouabain resistant (STO) feeder system and mitomycin-C-treated mouse embryonic fibroblast feeder system were used for SNUhES3, CHA3-hESC, and $\mathrm{H} 9$, respectively, in $0.1 \%$ gelatin (Sigma)- coated tissue culture dishes at $37^{\circ} \mathrm{C}$ and $5 \%$ $\mathrm{CO}_{2}$ in an air atmosphere. The medium for the three hESC lines, Dulbecco's modified Eagle's medium (DMEM)/F-12 (Invitrogen, Madison, WI), 20\% knock-out serum replacement (Invitrogen), $0.1 \mathrm{mM} \beta$-mercapto-ethanol (Sigma), 1\% nonessential amino acids (Gibco, Carlsbad, CA), $50 \mathrm{IU} / \mathrm{mL}$ penicillin, and $50 \mathrm{mg} / \mathrm{mL}$ streptomycin (Gibco), was supplemented with $0.4 \mathrm{ng} / \mathrm{mL}$ basic fibroblast growth factor (bFGF; Invitrogen) for SNUhES-3 cells, or $4 \mathrm{ng} / \mathrm{mL}$ bFGF for CHA3hESC and H9 cells, for the maintenance of undifferentiating hESCs, and it was changed daily. These cells were subcultured every 5 to 7 days by gently separating morphologically undifferentiated cells using a dissecting pipette.

\section{New EB-based method to derive MSCs from hESCs}

Derivation of MSCs from hESCs (hESC-MSCs) was achieved by multiple steps as described (Fig. 1a). (1) hESC colonies were detached after treatment with 0.5 unit $/ \mathrm{mL}$ Dispase (Gibco) for $30 \mathrm{~min}$. (2) The detached hESC colonies were incubated in bacterial dishes for 14 days to form EBs. The EB medium, hESC medium without bFGF, was changed every other day. (3) After selection of well-rounded EBs (about $250 \pm 50 \mu \mathrm{m}$ ) under the microscope, the EBs were reattached on a $0.1 \%$ gelatin-coated dish. The medium that was composed of DMEM low glucose (Gibco), 10\% fetal bovine serum (FBS) (Gibco), and 1\% antibiotic-antimycotic (Gibco) was changed every 3 days for 16 days. (4) The outgrowing cells from EBs were expanded by transfer to and culture in a $0.1 \%$ gelatin-coated dish with microvascular endothelial cell media-2 (EGM-2 MV) medium (Lonza, Basel, Switzerland). The derived cells were expanded by subculture for more than 150 days. Following the existing criteria, the cells were identified and named as hESC-MSCs; SNU3MSC, CHA3MSC, and H9MSC from SNUhES3, CHA3-hESC, and H9, respectively.

\section{Growth curve of hESC-MSCs}

A growth curve was established by multiplying the initial number of cells by the amplification fold for each passage.

\section{Test of teratoma formation by injection of hESC-MSCs to immunodeficient mice}

SNUhES3 $\left(3 \times 10^{6}\right)$ and hESC-MSCs derived from SNUhES3 (SNU3MSC-1, $1 \times 10^{7}$ ) were injected subcutaneously into the back of 5-week-old nonobese diabetic/severe combined immunodeficient (NOD/SCID) mice (JAX, Bar Harbor, ME). After 12 weeks, the mice were euthanized, and the resulting masses were removed. The excised mass was washed with phosphate-buffered saline (PBS) and fixed in $4 \% \mathrm{U}$ paraformaldehyde overnight at $4{ }^{\circ} \mathrm{C}$. Each tissue was embedded in paraffin or frozen in optimal cutting temperature (OCT) compound (Tissue-Teck; Sakura Finetek, Tokyo, Japan). Tissue sections $(7 \mu \mathrm{m})$ were stained with hematoxylin-eosin.

\section{Flow cytometry of hESC-MSCs}

For flow cytometry analysis, the cells were first dissociated by incubation at $37^{\circ} \mathrm{C}$ for $1 \mathrm{~min}$ in $0.25 \%$ trypsin/ ethylenediaminetetraacetic acid (Invitrogen), washed with PBS containing 2.5\% FBS, and incubated for $30 \mathrm{~min}$ with antibodies. Antibodies for hMSCs ${ }^{13,14}$ : CD29 (Phycoerythrin (PE); BD Biosciences, San Jose, CA), CD44 (Santacruz, Santa Cruz, CA), CD73 (PE; BD Biosciences), CD90 (Fluorescein isothiocyanate (FITC); BD Biosciences), and CD105 (FITC; Serotec, Raleigh, NC) for hESCs; SSEA1 (Santacruz), SSEA4 (e-Bioscience, San Diego, CA), OCT-4 (Chemicon, Temecula, CA), and TRA-1-60 (Chemicon) for other lineage markers; CD31 (PE; BD Biosciences), CD34 (Serotec), CD45 (Dako, Carpinteria, CA), and AC133 (PE; Miltenyi Biotec, Bergisch Gladbach, Germany) for immunogenic-related surface markers; human leukocyte antigen (HLA)-DR (FITC; BD Biosciences), HLA-DQ (FITC; BD Biosciences), B7-1 (FITC; BD Biosciences), and B7-2 (PE; BD Biosciences) were used. Appropriate secondary antibodies (Alexa 488 and 555, all from Invitrogen) were used to detect primary antibodies. After the treatment with antibodies, cells were analyzed with a flow cytometer (FACS calibur; BD Biosciences). Appropriate isotype controls were used for each antibody as a control for nonspecific antibody binding.

\section{Functional differentiation of hESC-MSCs to several lineages}

For adipocytic differentiation, hESC-MSCs were cultured in a differentiation medium containing alpha minimum essential medium (MEM) (Invitrogen), 10\% FBS (Gibco), $1 \mathrm{mM}$ dexamethasone, $10 \mu \mathrm{g} / \mathrm{mL}$ insulin, and $0.5 \mathrm{mM}$ isobutylxanthine (all from Sigma). The medium was changed every 3 days for 2 weeks. Adipocytes differentiated from hESC-MSCs were detected by Oil Red O staining. ${ }^{15}$

For chondrocytic differentiation, hESC-MSCs were formed into pellets and cultured in a differentiation medium containing high-glucose DMEM (Invitrogen), 10\% FBS (Gibco), $10 \mathrm{ng} / \mathrm{mL}$ transforming growth factor- $\beta 1$ (R\&D systems, Minneapolis, $\mathrm{MN}$ ), 1\% BD ${ }^{\mathrm{TM}}$ insulin, transferrin and selenium (ITS) + Premix (BD Bioscences, consisting of $6.25 \mu \mathrm{g} / \mathrm{mL}$ insulin, $6.25 \mu \mathrm{g} / \mathrm{mL}$ transferrin, $6.25 \mathrm{ng} / \mathrm{mL}$ selenious acid, $1.25 \mathrm{mg} / \mathrm{mL}$ serum albumin, and $5.35 \mu \mathrm{g} / \mathrm{mL}$ linoleic acid), $37.5 \mu \mathrm{g} / \mathrm{mL}$ ascorbate-2-phospate, $10^{-7} \mathrm{M}$ dexamethasone (all from Sigma), and 1\% nonessential amino acids (Invitrogen) for 3 weeks. Chondrocytes differentiated from hESCMSCs were stained with Alcian Blue. ${ }^{15}$ 


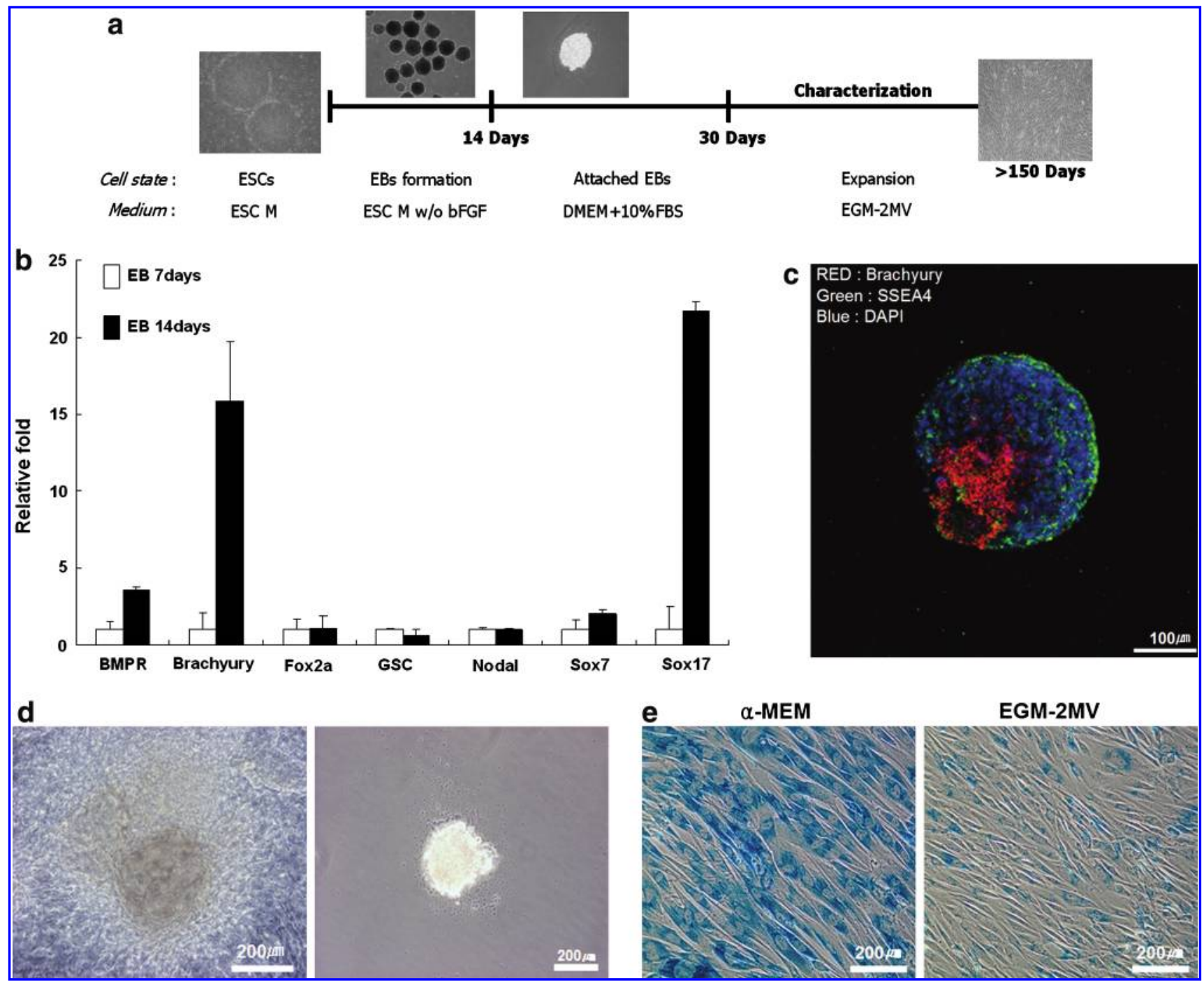

FIG. 1. Derivation of hMSCs from hESCs. (a) Experimental scheme for EB-based derivation of hESC-MSCs. Colonies of hESCs were enzymatically detached and then cultured in suspension condition to form EBs. After 14 days suspension culture, EBs were attached on plate and then attached EBs were cultured for following 16 days. Thereafter, hMSC looking cells outgrew from the attached EBs, and these cells were subcultured and expanded. (b) Expressions of mesoderm-specific genes in real-time polymerase chain reaction analysis are upregulated in EBs at day 14 of suspension culture compared with day 7. Each experiment was repeated twice, and the results were presented as mean \pm standard deviations. (c) EBs at day 14 showed expression of brachyury and SSEA4. (d) Some attached EBs produced outgrowing hESC-MSCs (day 30, left) and others went to apoptosis (right). (e) Beta-gal staining revealed that cells cultured in alpha-minimum essential medium ( $\alpha$-MEM) are prone to senescence than cells in EGM-2 MV. The cells obtained at day 95 were cultured for 1 month in $\alpha$-MEM or microvascular endothelial cell media-2 (EGM-2 MV). $\beta$-Galactosidase activity was detected at pH 6. ESC, embryonic stem cells; ESC M, ESC media; EB, embryoid body; hESC, human embryonic stem cell; hMSC, human mesenchymal stem cell. Color images available online at www.liebertonline.com/ten.

For osteocytic differentiation, hESC-MSCs were plated at low density in tissue culture dishes in a differentiation medium containing alpha MEM medium (Invitrogen), 10\% FBS (Gibco), $10 \mathrm{mM} \beta$-glycerol phosphate, $0.1 \mu \mathrm{M}$ dexamethasone, $200 \mu \mathrm{M}$ ascorbic acid (all from Sigma). The medium was changed every 3 to 4 days for 3 weeks. Osteocytes differentiated from hESCMSCs were stained using von Kossa staining. ${ }^{15}$

For myocytic differentiation, hESC-MSCs were cultured in alpha MEM medium (Invitrogen) with 20\% FBS (Gibco) for 3 weeks. Differentiated myocytes were stained using MyoD. ${ }^{14}$

\section{Real-time reverse transcription-polymerase} chain reaction analysis

Total RNAs from the cultured cells were extracted using the RNeasy Mini Kit (Qiagen, Valencia, CA) according to the manufacturer's protocol. cDNA was synthesized from approximately $1 \mu \mathrm{g}$ of total RNA using the Reverse Transcription System (Promega, Madison, WI) and subjected to polymerase chain reaction (PCR) amplification. The Primer3 software (Whitehead Institute/MIT Center for Genome Research) was used to design all the specific primers used 
Table 1. Real-Time Polymerase Chain Reaction Primer Sequences and Product Size

\begin{tabular}{|c|c|c|c|c|}
\hline \multirow{3}{*}{$\frac{\text { Genes }}{\text { Srebf1 }}$} & \multicolumn{2}{|c|}{ Primer } & \multirow{2}{*}{$\frac{\text { Product size }}{249}$} & \multirow{3}{*}{$\frac{\text { Temperature }}{60^{\circ} \mathrm{C}}$} \\
\hline & Forward & ggaaccatcttggcaacagt & & \\
\hline & Reverse & aatgtagtcgatggccttgc & & \\
\hline \multirow[t]{2}{*}{$P P A R \gamma$} & Forward & accaactttgggatcagctc & 244 & $60^{\circ} \mathrm{C}$ \\
\hline & Reverse & tctgcaaccactggatctgt & & \\
\hline \multirow{2}{*}{ Aggrecan } & Forward & aaaccacctctgcattccac & 211 & $60^{\circ} \mathrm{C}$ \\
\hline & Reverse & tctccgctgatttcagtcct & & \\
\hline \multirow[t]{2}{*}{$A L P$} & Forward & gaaccccaaaggcttcttct & 204 & $60^{\circ} \mathrm{C}$ \\
\hline & Reverse & ggggtgtatccaccaaatgt & & \\
\hline \multirow[t]{2}{*}{ Osteocalcin } & Forward & tgcagcetttgtgtccaa & 171 & $60^{\circ} \mathrm{C}$ \\
\hline & Reverse & tgaaagccgatgtggtca & & \\
\hline \multirow[t]{2}{*}{ Osteopontin } & Forward & gcaaccgaagttttcactcc & 176 & $60^{\circ} \mathrm{C}$ \\
\hline & Reverse & ccattcaactcctcgctttc & & \\
\hline \multirow[t]{2}{*}{ MyoD } & Forward & gcaggtgtaaccgtaaccca & 253 & $60^{\circ} \mathrm{C}$ \\
\hline & Reverse & cacacaccatgcctcagaga & & \\
\hline \multirow[t]{2}{*}{ GAPDH } & Forward & caacgaatttggctacagca & 177 & $60^{\circ} \mathrm{C}$ \\
\hline & Reverse & tgtgaggaggggagattca & & \\
\hline
\end{tabular}

in these experiments (Table 1). ${ }^{13}$ PCRs were performed using the ABI Prism 7000 sequence detection system (Applied Biosystems, Foster City, CA) with the SYBR ${ }^{\circledR}$ Green PCR Master Mix (Applied Biosystems). Glyceraldehyde 3-phosphate dehydrogenase (GAPDH) was simultaneously run as a control and used for normalization. Nontemplate control wells without cDNA were included as negative controls. Each test sample was run in duplicate. The results are reported as relative expressions after normalization of transcript amount to the endogenous control using the $2^{-\Delta \Delta C_{T}}$ method. ${ }^{16}$ The threshold cycle $\left(C_{\mathrm{T}}\right)$ indicates the fractional cycle number at which the amount of amplified target reaches a fixed threshold.

The following formula was used for the analysis.

Gene expression level $=2^{-\Delta \Delta C_{T}}$.

\section{Myocardial cryoinjury model and hESC-MSCs transplantation}

All animal experiments were performed after receiving approval from the Institutional Animal Care and Use Committee of the Clinical Research Institute in Seoul National University Hospital and complied with the National Research Council's Guidelines for the Care and Use of Laboratory Animals (revised 1996). C57BL/6J mouse was anesthetized with tiletamine with diazepine (Zoletil, $25 \mathrm{mg} / \mathrm{kg}$; Virbac, Fort Worth, TX) plus Xylazine $(10 \mathrm{mg} / \mathrm{kg}$; Bayer, North Rhine-Westphalia, Germany) by intramuscular injection. Experimental myocardial cryoinjury, modified from previous study, ${ }^{17}$ was produced by freeze-thaw technique with application of precooled cryoinjury probe $(4 \mathrm{~mm}$ diameter) on anterior left ventricular wall for $25 \mathrm{~s}$. C57BL/6J mouse was randomized into media injection group (control) and cell injection group. For cell transplantation group, a total of $5 \times 10^{4}$ SNU3MSC- $1 / 60 \mu \mathrm{L} 0.9 \%$ saline was transplanted by two intramyocardial injections.

\section{Echocardiographic evaluation}

Transthoratic echocardiography was performed 3 days after cryoinjury for baseline evaluation, and then 4 and 8 weeks after cryoinjury with an echocardiographic system (Aplio XG, Toshib, Japan) equipped with a 15-MHz lineararray transducer. Left ventricular end-diastolic dimensions (LVEDD) and left ventricular end-systolic dimensions were measured according to the leading edge method of the American Society of Echocardiography. ${ }^{18}$ The percent left ventricular fractional shortening was calculated as $100 \times$ (LVEDD - left ventricular end-systolic dimensions)/LVEDD.

\section{Histological preparations and analysis}

After echocardiographic evaluation, mice were euthanized, and the hearts were removed. The excised heart was retrograde perfused with PBS for coronary vasculature and left ventricular washing, and fixed with $4 \%$ paraformaldehyde overnight at $4{ }^{\circ} \mathrm{C}$ and then $15 \%$ sucrose for overnight at $4{ }^{\circ} \mathrm{C}$. Each tissue was embedded in paraffin. Section $(7 \mu \mathrm{m})$ was stained with Masson's trichrome to detect infarct area and calculated using Image-Pro plus 4.5 software.

\section{Statistical analysis}

Data are presented as mean \pm standard deviation. Continuous variables are compared between groups using student's $t$-test. Analysis was performed by SPSS 12.0. A probability value of $<0.05$ was considered statistically significant.

\section{Results}

\section{EB-based derivation methods of hESC-MSCs}

hESC-MSC lines were derived from three different hESCs by EB-based differentiation methods. Derivation methods can be summarized by the following four steps (Fig. 1a): (1) enzymatic detachment of hESC colonies, (2) formation of EBs from suspension culture of hESCs for 14 days, (3) reattachment of EBs on plate and culture of outgrowing cells, and then (4) expansion of the outgrowing hMSCs. To determine the optimal timing of reattaching EBs on plate after the formation of EBs from suspension culture, we compared the expression of mesoderm marker genes in EBs at days 7 and 14 of suspension culture, using real-time PCR. Expressions of BMPR2, Brachyury, and Sox17 of EBs are higher 

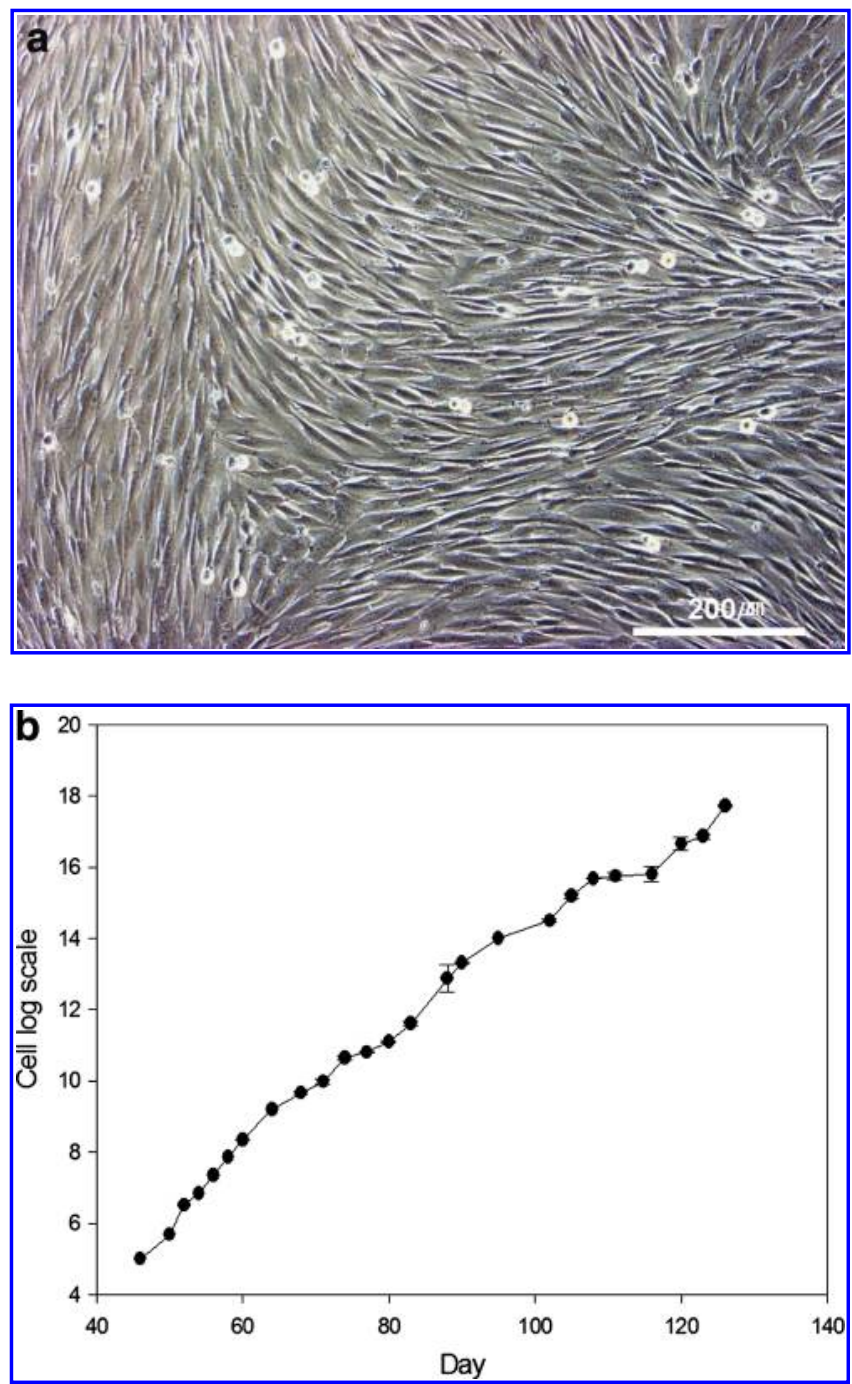

FIG. 2. Morphology and growth curve of hESC-MSCs. (a) Morphology of SNU3MSC-1 at confluence under phasecontrast microscopy exhibits a typical fingerprint-like pattern. (b) SNU3MSC-1 was subcultured every 3-4 days over 120 days. Color images available online at www.liebertonline .com/ten.

at day 14 of suspension culture than day 7 (Fig. 1b, c). Timing of reattachment was determined based on the level of mesoderm marker expression in EBs and yield of outgrowing hESC-MSCs from the attached EBs. Around 16 days after reattachment, EBs went different courses; some EBs produced the outgrowing cells, whereas the others went to apoptosis (Fig. 1d). At this time (day 30 from the start of EBs suspension culture), the cells outgrowing from EBs were transferred to a $0.1 \%$ gelatin-coated tissue culture dish in EGM-2 MV medium. EGM-2 MV medium prevents senescence of outgrowing cells during the long-term cultivation compared with $\alpha$-MEM (Fig. 1e), but the underlying mechanism is still unclear. Outgrowing cells, named hESCMSCs, could be stably expanded by subcultures and showed uniform characteristic morphology of hMSCs. Proliferative capacity of hESC-MSCs was maintained till 35 passages (Fig. 2).

\section{Characterization of hESC-MSCs}

The three different hESC lines of SNUhES3, CHA3-hESCs, and $\mathrm{H} 9$ were used in this study to derive hESC-MSC lines. hMSCs derived from SNUhES3, CHA3-hESCs, and H9 were named SNU3MSC-1 5, CHA3MSC-1 2, and H9MSC-1 2 respectively (Table 2). After the derivation of hESC-MSCs, characteristics of derived cells were identified by the typical morphology, cell surface markers, and proliferative and differentiation potentials into mesenchymal lineages such as adipocyte, chondrocyte, and osteocyte. ${ }^{13}$

SNU3MSC-1 expressed CD29, CD44, CD73, CD90, and CD105 (Fig. 3). Expressions of CD73 and CD105 were gradually increased as passages went. SNU3MSC-1 did not express hESC-specific marker; SSEA1, SSEA4, TRA-1-60, and OCT-4 after 95 days from start of EBs suspension culture. These results suggested that contamination of hESCs is minimized, and the risk of tumorigenicity also can be minimized in SNU3MSC-1. SNU3MSC-1 was also negative for other lineage markers of CD31, CD34, CD45, and AC133 (Fig. 3b). Other hESC-MSC lines, CHA3MSC-1 and H9MSC1 , also showed similar patterns of marker expression and morphology to SNU3MSC-1, which suggests EB-based hESC-MSCs derivation method can be reproducibly applied to CHA3-hESCs and H9 (Supplemental Fig. 1A-D available online at www.liebertonline.com/ten).

To prove the multipotency of SNU3MSC-1, we tried to induce SNU3MSC-1 to differentiate into adipogenic, chondrogenic, osteogenic, and myogenic lineage under specialized culture conditions. After 14 to 21 days of culture in the adipogenic differentiation medium, fat granules appeared and grew in size (Fig. 4a). Expression of adipocyte-specific markers of PPAR $\gamma$ and Srebf1 also increased in real-time PCR (Fig. 4b). Chondrogenic differentiation of SNU3MSC-1 was induced using the pellet culture method. After 3 weeks of induction, more than $90 \%$ of all cells stained positive with Alcian Blue, a specific marker for extracellular matrix proteoglycans (Fig. 4a). The expression

Table 2. Three Different Human Embryonic Stem Cell Lines Used and Human Embryonic Stem Cell-Mesenchymal Stem Cell Lines Established in This Study

\begin{tabular}{|c|c|c|c|c|}
\hline \multirow[b]{2}{*}{ ESC line } & \multicolumn{2}{|c|}{ Culture condition } & \multirow[b]{2}{*}{ Race/sex } & \multirow[b]{2}{*}{ Established hESC-MSCs } \\
\hline & Feeder type & bFGF con. $(n g / m L)$ & & \\
\hline SNUhES3 & STO & 0.4 & Oriental/XY & SNU3MSC-1 5 \\
\hline CHA3-hESC & MEF & 4 & Oriental/XY & CHA3MSC-1, 2 \\
\hline H9 & MEF & 4 & Caucasian/XX & H9MSC-1, 2 \\
\hline
\end{tabular}

bFGF, basic fibroblast growth factor; hESC, human embryonic stem cell; MEF, mouse embryonic fibroblast. 


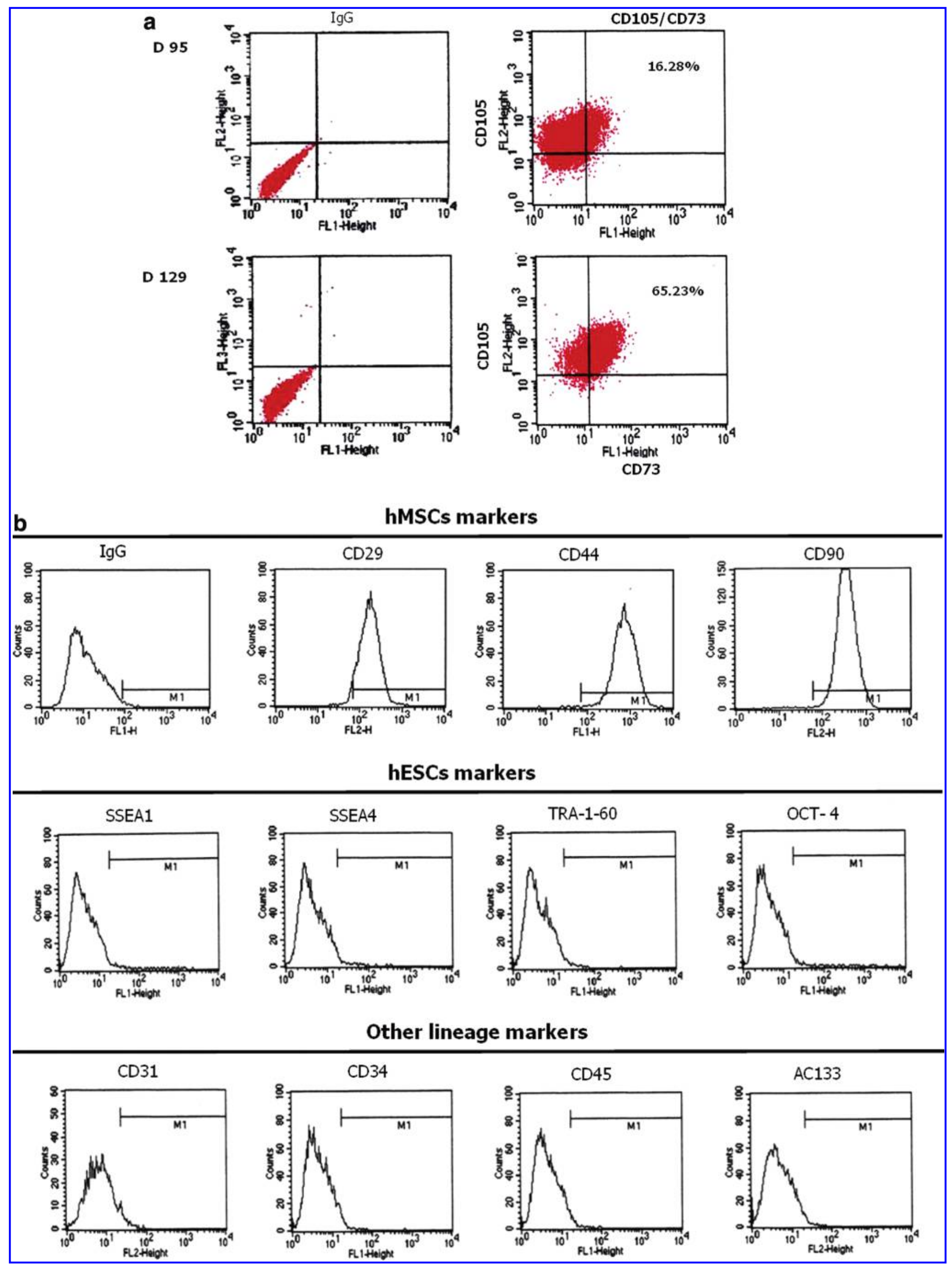



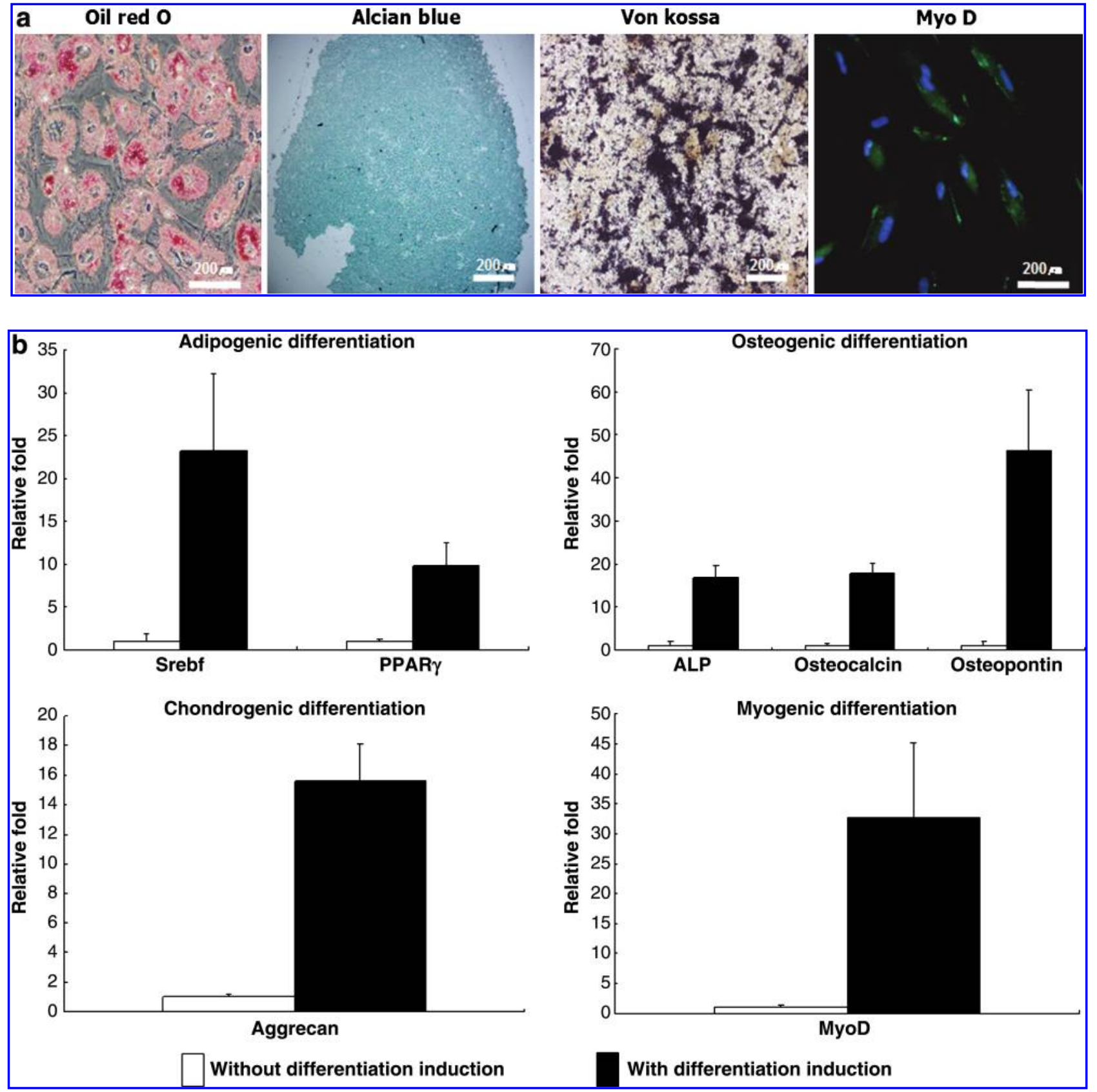

FIG. 4. Functional differentiation of hESC-MSCs into several lineages. (a) SNU3MSC-1 differentiated into adipocytes, chondrocytes, osteocytes, and myocytes by culture in specified differentiation media, respectively. (b) Lineage-specific gene expression was determined by real-time polymerase chain reaction. Srebf1 and PPAR $\gamma$ for adipocytes; aggrecan for chondrocytes; ALP, osteocalcin, and osteopontin for osteocytes; and MyoD for myocytes. Each experiment was repeated twice, and the results were presented as mean \pm standard deviations. Color images available online at www.liebertonline.com/ten.

of chondrogenic gene such as aggrecans, which are components of the extracellular matrix selectively expressed in chondrocytes, was confirmed by real-time PCR (Fig. 4b). Osteogenic differentiation was observed in the presence of $\beta$-glycerol phosphate. After 3 weeks of induction, calcium deposits were observed in the matrix and stained with von Kossa (Fig. 4a). The gene levels of ALP, osteocalcin, and osteopontin, which are bone specific markers, also increased (Fig. 4b). Additionally, myogenic differentiation of SNU3MSC-1 was successfully achieved using alpha MEM

FIG. 3. Flow cytometry analysis of SNU3MSC-1. After expansion for 95 and 129 days, cells were trypsinized and stained with specific markers for hMSCs (CD73, CD105) (a), hESCs surface markers, and other lineage markers (b). They were positive for hMSC markers, whereas negative for hESCs or other lineage markers. Color images available online at www.liebertonline.com/ten. 

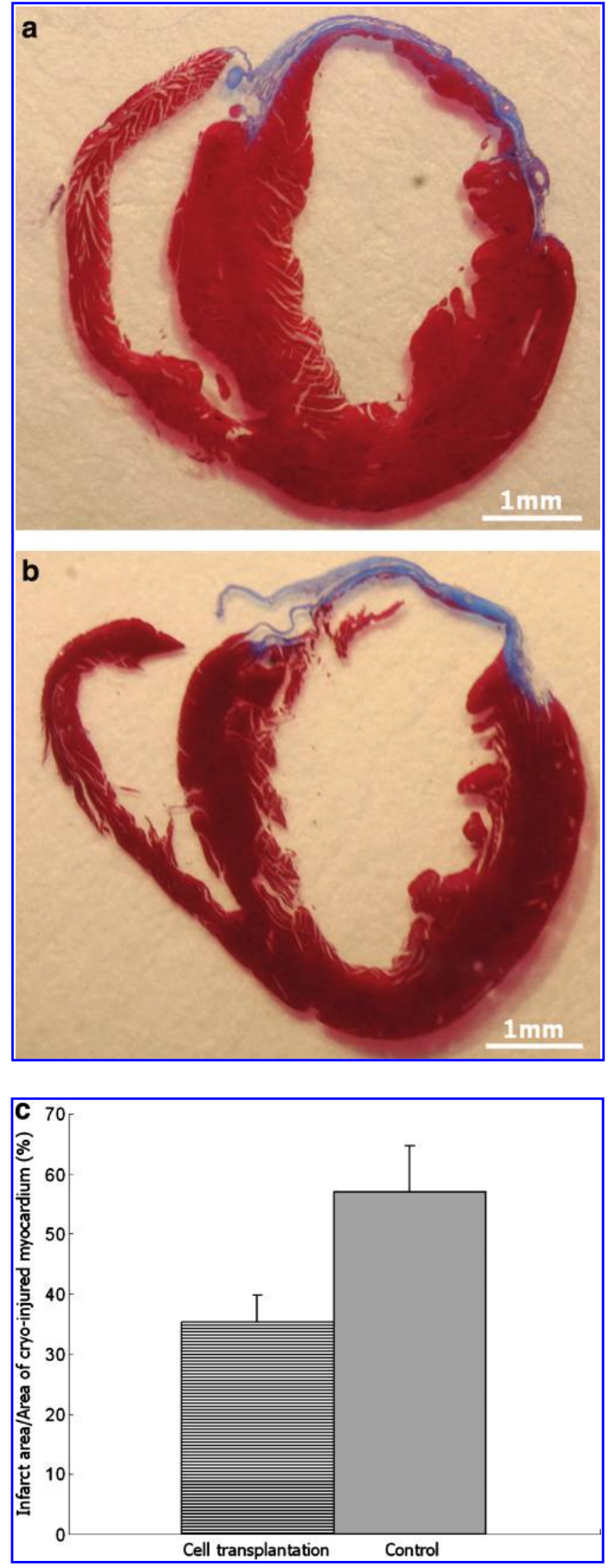

medium with $20 \%$ heat-inactivated FBS. After 2 weeks of induction, myocyte-like cells appeared and expressed MyoD (Fig. 4). From these results, we can conclude that hESC-MSCs have differentiation potential to multiple mesenchymal derivatives.

\section{Feasibility in therapeutic application}

In myocardial cryoinjury model, mice which received transplantation of SNU3MSC-1 showed significantly better cardiac function measured by echocardiography compared with the injury-only group at 4 weeks after transplantation (left ventricular fractional shortening at 4 weeks in control $[n=5]$ vs. cell transplantation group [ $n=5]: 20.3 \pm 2.9 \%$ vs. $25.0 \pm 2.2 \%, p<0.05)$. Although statistical significance of difference in cardiac function between groups was not maintained till 8 weeks $(20.5 \pm 1.0 \%$ vs. $23.7 \pm 3.7 \%, p=0.097$, Table $3)$, cell transplantation showed smaller infarct in injured area compared with the control group at 8 weeks $(35.5 \pm 4.4 \%$ vs. $57.0 \pm 7.8 \% ; p<0.001$, Fig. 5a-c).

\section{Safety and stability of hESC-MSCs in therapeutic application}

To investigate the safety and stability of hESC-MSCs for therapeutic application, we performed karyotyping, in vivo teratoma formation assay, and fluorescence-activated cell sorting (FACS) analysis of immune-related surface markers. After prolonged culture up to 161 days, SNU3MSC-1 showed normal karyotype identical to that of the original hESCs (Fig. 6a). CHA3MSC-1 and H9MSC-1 also showed normal karyotype after long-term culture (Supplemental Fig. $1 \mathrm{E}, \mathrm{F})$. These results confirmed the chromosomal stability of hESC-MSCs in long-term cultivation. We evaluated tumorigenicity of hESC-MSCs (SNU3MSC-1) in NOD/SCID mice in comparison to original hESC line (SNUhES3). We observed that SNUhES3 formed teratoma containing three-germ layer derivatives in NOD/SCID mice (Fig. 6b). In contrast, SNU3MSC-1 did not form teratoma or any tumor after transplantation of threefold higher number of cells than hESCs, which is significantly higher than the threshold dose that was required to form teratoma. ${ }^{19}$

Moreover, SNU3MSC-1 showed immunotolerant phenotypes like other hMSCs obtained from adults in FACS analysis (Fig. 6c). Expressions of HLA-DR, DQ, and costimulators B-7.1 and B-7.2 were not detected on the surface of SNU3MSC-1. In previous reports, maintenance with bFGF resulted in increased HLA-DR expression in hMSCs. ${ }^{20}$ But, SNU3MSC-1 did not express HLA-DR, HLA-DQ, and costimulators during and after long-term culture with bFGFcontaining culture media. Further, SNU3MSC-1 showed stable and constant immunophenotypes even after multiple cycles of freezing-thawing.

FIG. 5. Therapeutic potentials in mice myocardial cryoinjury model. The mice received SNU3MSC-1 transplantation (a) showed smaller infarct size compared with the control mouse (b) at 8 weeks after transplantation. Each slide was stained with Masson's trichrome. (c) Infarct area (blue) was calculated by Image-Pro plus 4.5 software (cell transplantation; $35.5 \pm 4.4 \%$ vs. control; $57.0 \pm 7.8 \%$; $p<0.001$ ). Color images available online at www.liebertonline.com/ten. 


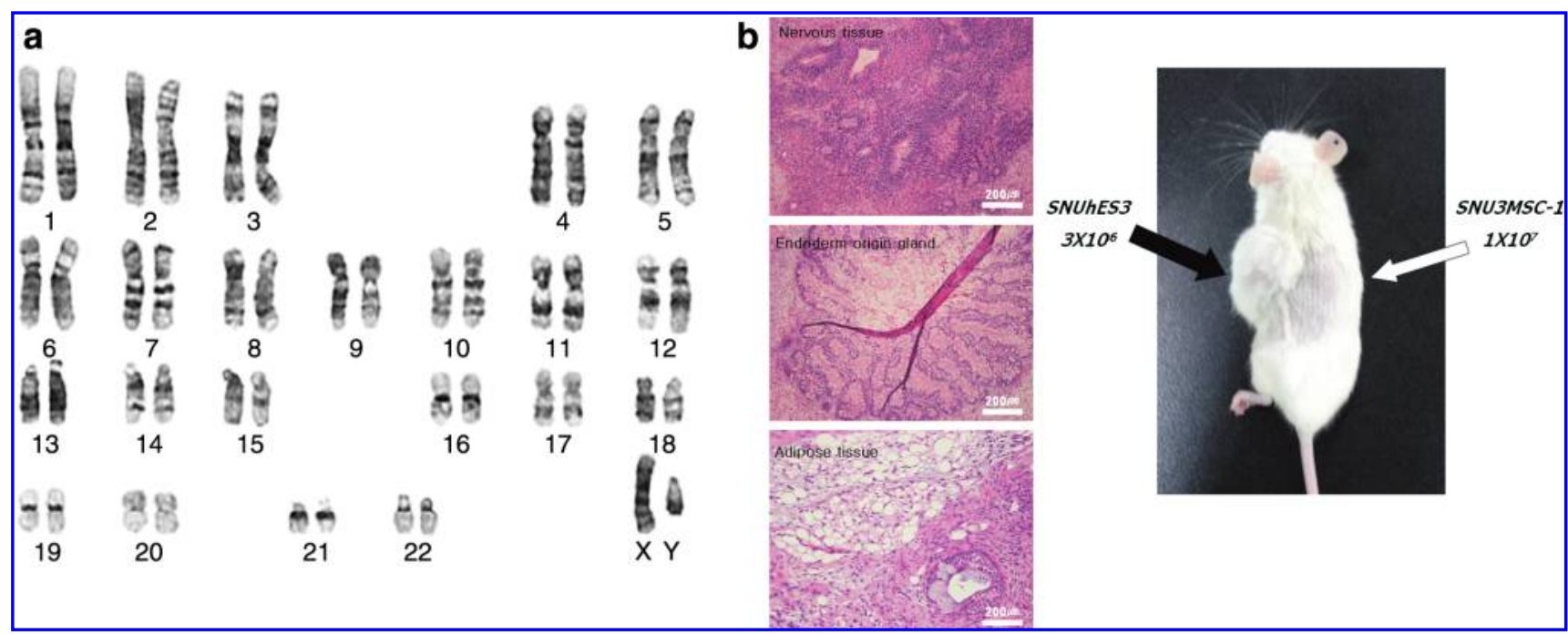

FIG. 6. Karyotyping, tumorigenic potentials, and immunophenotypes. (a) SNU3MSC-1 maintained normal karyotype after 161 days of culture in expansion conditions, which was proved by G-band staining. (b) Hematoxylin-eosin staining: the potential of teratocarcinoma formation was tested by injection of cells into nonobese diabetic/severe combined immunodeficient mice. Aliquots of $1 \times 10^{7}$ and $3 \times 10^{6}$ cells of SNU3MSC-1 and SNUhES3, respectively, were injected. Although SNUhES3 formed teratocarcinoma, SNU3MSC-1 did not produce tumor. (c) Expression of human leukocyte antigen (HLA) type II and costimulators were not observed in SNU3MSC-1 by FACS analysis after 95 and 129 days of cultivation. Color images available online at www.liebertonline.com/ten.

(Continued $\rightarrow$ )

\section{Discussion}

In this study, we established a novel, simplified derivation method of hESC-MSCs. Our EB-based derivation method is reproduced in three different hESC lines. We also proved the therapeutic efficacy of derived hESC-MSCs in mice myocardial cryoinjury model. Our protocol did not require mechanical selection and separation.

Several derivation methods of MSCs from hESCs were reported recently. ${ }^{8-10}$ These reports bear some problems such as the risk of animal cell or pathogen contamination, ${ }^{8}$ the limited differentiation potency of derived cells, ${ }^{9}$ and the requirement of FACS sorting that has risks of damaging the cells. 8,10 In addition, these methods used only one or two hESC lines in their experiments. Our derivation protocol has several advantages compared to the previously reported methods.

First, our protocol has a potential to be a standard derivation method that can be generally be applicable to various hESC lines. Previous protocols may have forced researchers to develop customized derivation protocols for each hESCs. The process is time- and labor consuming. In this study we attempted to develop a generally applicable method to derive hMSCs from hESCs and successfully derived hESC-MSC lines from three different hESCs: SNUhES3, CHA3-hESCs, and H9. These three hESCs are derived from donors of different races and sexes (Table 2). However, our simple deri- vation protocol reproducibly established hESC-MSCs without individualized modification for each hESC line.

Second, hESC-MSCs obtained by our protocol have shown the genetic stability and biologic safety. Although we could not evaluate possibility of gene translocation in this study, we did not observe any chromosomal abnormality with Gbanding in all hESC-MSC lines even after multiple cycles of freezing-thawing. Additionally, they did not provoke a tumor in NOD/SCID mice. Previous studies reported tumorigenic risk of cells derived from hESCs. Even the terminally differentiated cells derived from hESCs such as cardiomyocytes still have the possible risk of tumorigenicity. ${ }^{21}$ In our study even though significantly higher numbers of hESC-MSCs than previously reported tumorigenic threshold were transplanted in NOD/SCID mice, ${ }^{19}$ we did not observe the formation of tumors. Moreover, our protocol did not require xenogenic feeder. Xenogenic feeder is one of the major limiting factors for the application of hESCs to clinical field as they possess the risk of transmitting pathogens and other unidentified risks. ${ }^{10}$

Third, our hESC-MSCs have powerful self-renewal and proliferative capacities. They can be stably cultured and expanded at least up to 35 passages and after multiple freezethaw cycles. It makes possible to obtain and store sufficient amounts of hESC-MSCs from a single derivation. The derived cells in turn can be prepared and stored by HLA type

Table 3. Echocardiographic Variables in Mice Cryoinjury Model

\begin{tabular}{|c|c|c|c|c|c|c|}
\hline & \multicolumn{2}{|c|}{ Baseline } & \multicolumn{2}{|c|}{4 weeks } & \multicolumn{2}{|c|}{8 weeks } \\
\hline & LVEDD $(\mathrm{mm})$ & LVFS (\%) & LVEDD (mm) & LVFS (\%) & LVEDD $(\mathrm{mm})$ & LVFS $(\%)$ \\
\hline Control & $39.1 \pm 3.8$ & $22.1 \pm 0.2$ & $41.6 \pm 3.7$ & $20.3 \pm 2.9$ & $42.6 \pm 4.7$ & $20.5 \pm 1.0$ \\
\hline Cell transplantation & $38.0 \pm 0.9$ & $22.8 \pm 0.4$ & $40.0 \pm 1.5$ & $25.0 \pm 2.2$ & $41.1 \pm 2.5$ & $23.7 \pm 3.7$ \\
\hline
\end{tabular}

LVEDD, left ventricular end-diastolic dimension; LVFS, left ventricular fractional shortening. 


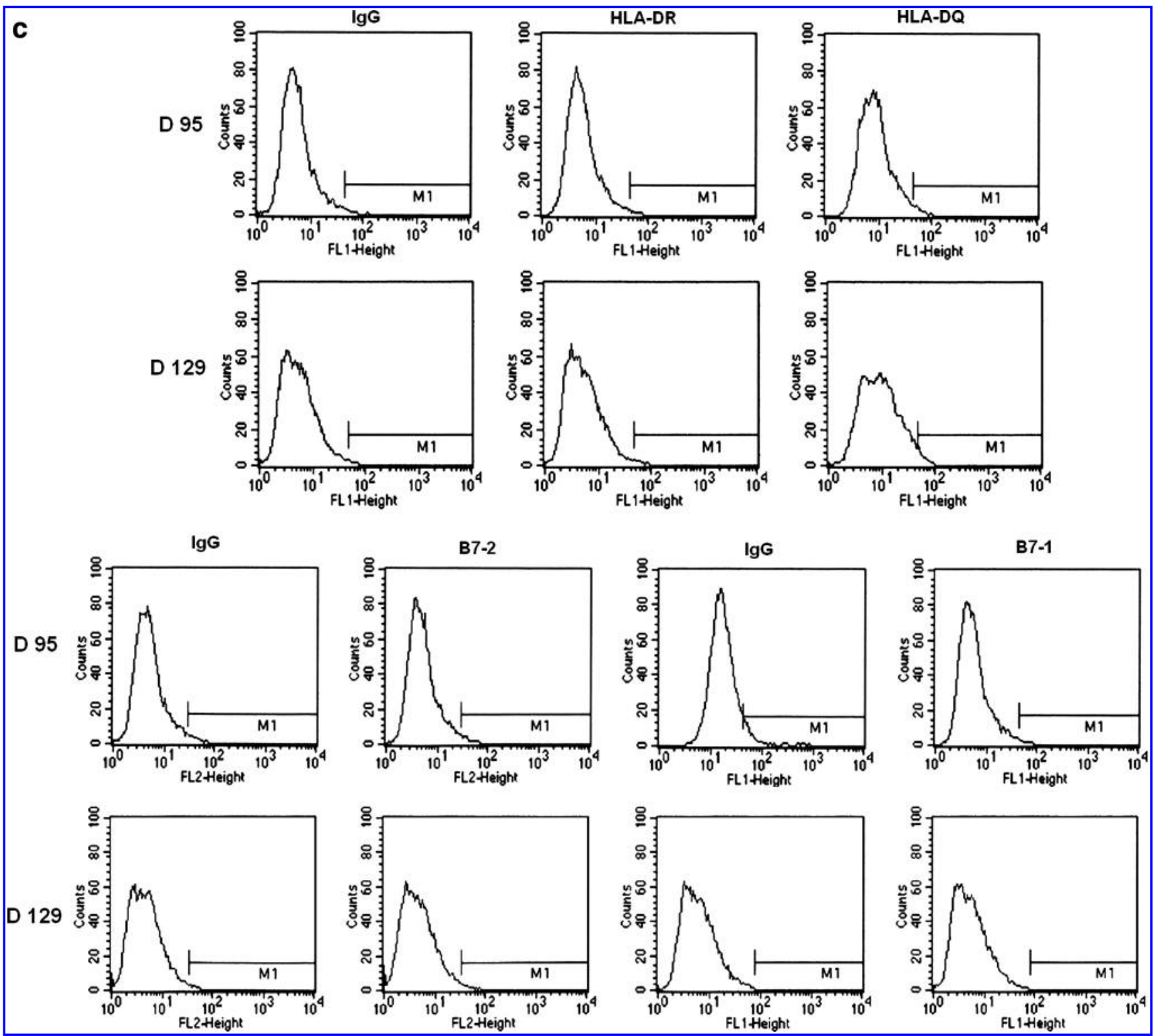

FIG. 6. (Continued).

to satisfy future demands and thus serve well as off the shelf allogenic stem-cell sources. Although relatively longer derivation time than previously reported protocols can be a limitation of our protocol, expansibility and storability of hESC-MSCs obtained by our protocol offset the potential problems related with longer derivation time.

Fourth, our hESC-MSCs have potentials to obtain immune privileges like other hMSCs. ${ }^{7}$ In small pilot study using myocardial cryoinjury, improvement of cardiac function after SNU3MSC-1 transplantation is comparable between immunocompetent mice and athymic nude mice. These results suggest that SNU3MSC-1 is immunetolerant in vivo model like most of the adult MSCs. Immune tolerability of hMSCs makes HLA-mismatched allogenic hMSC transplantation possible in clinical trials. Our hESC-MSCs did not express HLA-DR, DQ, and costimulators B-7.1 and B-7.2. Nonexpression of these surface antigens might contribute to immune tolerability of hESC-MSCs. However, it should be confirmed in the future studies.
In the future clinical application of hESC-MSCs, the clonal derivation from single cells is very useful. However, most reported hESC lines are not clonally derived and also have a potential to contain multiple precursor cells that are committed to certain lineages. In addition, several hESC lines are reported to develop abnormal karyotype under certain culture conditions. Recently, Heins et al. reported clonal derivation of hESC line that had karyotype of trisomy. ${ }^{22}$ Although we could not detect abnormal karyotype in hESC lines used, we cannot clearly exclude the possibility of heterogeneity of original cells. For that reason, we tried clonal derivation from early outgrowing cells at protocol day 40 without selection by a specific marker. However, in this experiment we failed to achieve consistent clonal expansion of hESC-MSCs from a single cell. In animal study, we observed improvement of cardiac function in cell transplantation group. However, improvement of cardiac function in cell-transplanted group could not be maintained till later phase of observation. Mechanism of hESC-MSCs func- 
tionality in in vivo model needs to be analyzed in further studies.

In summary, our new EB-based method to derive hMSCs from hESCs is characterized as generalized applicability and safety. This protocol will provide a more effective platform for the production of large amount of genetically identical hMSCs from each hESC line.

\section{Acknowledgment}

This study was supported by a grant for Stem Cell Research Center (SC4210).

\section{Disclosure Statement}

The authors have no conflicts of interest.

\section{References}

1. Rando, T.A. Stem cells, ageing and the quest for immortality. Nature 441, 1080, 2006.

2. Kang, S.M., Cho, M.S., Seo, H., Yoon, C.J., Oh, S.K., Choi, Y.M., and Kim, D.W. Efficient induction of oligodendrocytes from human embryonic stem cells. Stem Cells 25, 419, 2007.

3. D'Amour, K.A., Agulnick, A.D., Eliazer, S., Kelly, O.G., Kroon, E., and Baetge, E.E. Efficient differentiation of human embryonic stem cells to definitive endoderm. Nat Biotechnol 23, 1534, 2005.

4. Burridge, P.W., Anderson, D., Priddle, H., Barbadillo Muñoz, M.D., Chamberlain, S., Allegrucci, C., Young, L.E., and Denning, C. Improved human embryonic stem cell embryoid body homogeneity and cardiomyocyte differentiation from a novel V-96 plate aggregation system highlights interline variability. Stem Cells 25, 929, 2007.

5. Brederlau, A., Correia, A.S., Anisimov, S.V., Elmi, M., Paul, G., Roybon, L., Morizane, A., Bergquist, F., Riebe, I., Nannmark, U., Carta, M., Hanse, E., Takahashi, J., Sasai, Y., Funa, K., Brundin, P., Eriksson, P.S., and Li, J.Y. Transplantation of human embryonic stem cell-derived cells to a rat model of Parkinson's disease: effect of in vitro differentiation on graft survival and teratoma formation. Stem Cells 24, 1433, 2006.

6. Fujikawa, T., Oh, S.H., Pi, L., Hatch, H.M., Shupe, T., and Petersen, B.E. Teratoma formation leads to failure of treatment for type I diabetes using embryonic stem cell-derived insulin-producing cells. Am J Pathol 166, 1781, 2005.

7. Aggarwal, S., and Pittenger, M.F. Human mesenchymal stem cells modulate allergenic immune cell responses. Blood 105, 1815, 2005.

8. Barberi, T., Willis, L.M., Socci, N.D., and Studer, L. Derivation of multipotent mesenchymal precursors from human embryonic stem cells. PLoS Med 2, 554, 2005.

9. Oliver, E.N., Rybicki, A.C., and Bouhassira, E.E. Differentiation of human embryonic stem cells into bipotent mesenchymal stem cells. Stem cells 24, 1914, 2006.

10. Lian, Q., Lye, E., Suan Yeo, K., Khia Way Tan, E., SaltoTellez, M., Liu, T.M., Palanisamy, N., El Oakley, R.M., Lee, E.H., Lim, B., and Lim, S.K. Derivation of clinically compliant MSCs from CD105+, CD24- differentiated human ESCs. Stem cells 25, 425, 2007.

11. Oh, S.K., Kim, H.S., Ahn, H.J., Seol, H.W., Kim, Y.Y., Park, Y.B., Yoon, C.J., Kim, D.W., Kim, S.H., and Moon, S.Y. Derivation and characterization of new human embryonic stem cell lines: SNUhES1, SNUhES2, and SNUhES3. Stem Cells 23, 211, 2005.

12. Kim, S., Ahn, S.E., Lee, J.H., Lim, D.S., Kim, K.S., Chung, H.M., and Lee, S.H. A novel culture technique for human embryonic stem cells using porous membranes. Stem Cells 25, 2601, 2007.

13. Pittenger, M.F., Mackay, A.M., Beck, S.C., Jaiswal, R.K., Douglas, R., Mosca, J.D., Moorman, M.A., Simonetti, D.W., Craig, S., and Marshak, D.R. Multilineage potential of adult human mesenchymal stem cells. Science 284, 143, 1999.

14. Dezawa, M., Ishikawa, H., Itokazu, Y., Yoshihara, T., Hoshino, M., Takeda, S., Ide, C., and Nabeshima, Y. Bone marrow stromal cells generate muscle cells and repair muscle degeneration. Science 309, 314, 2005.

15. Lee, R.H., Kim, B., Choi, I., Kim, H., Choi, H.S., Suh, K., Bae, Y.C., and Jung, J.S. Characterization and expression analysis of mesenchymal stem cells from human bone marrow and adipose tissue. Cell Physiol Biochem 14, 311, 2004.

16. Livak, K.J., and Schmittgen, T.D. Analysis of relative gene expression data using real-time quantitative PCR and the $22^{-\Delta \Delta C_{T}}$ method. Methods 25, 402, 2001.

17. Soonpaa, M.H., Koh, G.Y., Klug, M.G., and Field, L.J. Formation of nascent intercalated disks between grafted fetal cardiomyocytes and host myocardium. Science 264, 98, 1994.

18. Murphy, J.G., and Lloyd, M.A. Mayo Clinic Cardiology, third edition. Mayo Clinic Scientific Press, Informa Healthcare, 2006.

19. Nussbaum, J., Minami, E., Laflamme, M.A., Virag, J.A., Ware, C.B., Masino, A., Muskheli, V., Pabon, L., Reinecke, H., and Murry, C.E. Transplantation of undifferentiated murine embryonic stem cells in the heart: teratoma formation and immune response. FASEB J 21, 1345, 2007.

20. Sotiropoulou, P.A., Perez, S.A., Salagianni, M., Baxevanis, C.N., and Papamichail, M. Characterization of the optimal culture conditions for clinical scale production of human mesenchymal stem cells. Stem Cells 24, 462, 2005.

21. Leor, J., Gerecht, S., Cohen, S., Miller, L., Holbova, R., Ziskind, A., Shachar, M., Feinberg, M.S., Guetta, E., and Itskovitz-Eldor, J. Human embryonic stem cell transplantation to repair the infarcted myocardium. Heart 93, 1278, 2007.

22. Heins, N., Lindahl, A., Karlsson, U., Rehnström, M., Caisander, G., Emanuelsson, K., Hanson, C., Semb, H., Björquist, P., Sartipy, P., and Hyllner, J. Clonal derivation and characterization of human embryonic stem cell lines. I Biotechnol 122, 511, 2006.

Address correspondence to: Hyo-Soo Kim, M.D.

Department of Internal Medicine Seoul National University Hospital 28 Yongon-dong Chongno-gu

Seoul 110-744

Republic of Korea

E-mail: hyosoo@snu.ac.kr

Hyun-Jae Kang, M.D. Department of Internal Medicine Seoul National University Hospital 28 Yongon-dong

Chongno-gu

Seoul 110-744 Republic of Korea

E-mail: nowkang@snu.ac.kr

Received: October 27, 2008

Accepted: September 21, 2009

Online Publication Date: November 3, 2009 



\section{This article has been cited by:}

1. Eun Ju Lee, Hyun-Jae Kang, Ha-Neul Lee, Soo Kyung Kang, Keum-Hyun Kim, Sae-Won Lee, Gene Lee, Young-Bae Park, Hyo-Soo Kim. 2012. New culture system for human embryonic stem cells: Autologous mesenchymal stem cell feeder without exogenous fibroblast growth factor 2. Differentiation 83:1, 92-100. [CrossRef]

2. Eun Ju Lee, Eue-Keun Choi, Soo Kyoung Kang, Gi-Hwan Kim, Ju Young Park, Hyun-Jae Kang, Sae-Won Lee, Keum-Hyun Kim, Jin Sook Kwon, Ki Hong Lee, Youngkeun Ahn, Ho-Jae Lee, Hyun-Jai Cho, Soo Jin Choi, Won Il Oh, Young-Bae Park, Hyo-Soo Kim. 2011. N-cadherin Determines Individual Variations in the Therapeutic Efficacy of Human Umbilical Cord Blood-derived Mesenchymal Stem Cells in a Rat Model of Myocardial Infarction. Molecular Therapy . [CrossRef]

3. Ivana Gadjanski, Kara Spiller, Gordana Vunjak-Novakovic. 2011. Time-Dependent Processes in Stem Cell-Based Tissue Engineering of Articular Cartilage. Stem Cell Reviews and Reports . [CrossRef]

4. Rongrong Wu, Bin Gu, Xiaoli Zhao, Zhou Tan, Liangbiao Chen, Jiang Zhu, Ming Zhang. 2011. Derivation of multipotent nestin+/CD271-/STRO-1- mesenchymal-like precursors from human embryonic stem cells in chemically defined conditions. Human Cell . [CrossRef]

5. Wei Seong Toh, Eng Hin Lee, Tong Cao. 2010. Potential of Human Embryonic Stem Cells in Cartilage Tissue Engineering and Regenerative Medicine. Stem Cell Reviews and Reports . [CrossRef]

6. Rachel A Oldershaw, Melissa A Baxter, Emma T Lowe, Nicola Bates, Lisa M Grady, Francesca Soncin, Daniel R Brison, Timothy E Hardingham, Susan J Kimber. 2010. Directed differentiation of human embryonic stem cells toward chondrocytes. Nature Biotechnology 28:11, 1187-1194. [CrossRef]

7. Yi-Chun Yeh, Wen-Yu Lee, Chu-Leng Yu, Shiaw-Min Hwang, Min-Fan Chung, Li-Wen Hsu, Yen Chang, Wei-Wen Lin, Ming-Song Tsai, Hao-Ji Wei, Hsing-Wen Sung. 2010. Cardiac repair with injectable cell sheet fragments of human amniotic fluid stem cells in an immune-suppressed rat model. Biomaterials 31:25, 6444-6453. [CrossRef]

8. D. Evseenko, Y. Zhu, K. Schenke-Layland, J. Kuo, B. Latour, S. Ge, J. Scholes, G. Dravid, X. Li, W. R. MacLellan, G. M. Crooks. 2010. Mapping the first stages of mesoderm commitment during differentiation of human embryonic stem cells. Proceedings of the National Academy of Sciences 107:31, 13742-13747. [CrossRef] 